\title{
Uusioersily
}

\section{The DSM-5 dissociative-PTSD subtype: Can levels of depression, anxiety, hostility, and sleeping difficulties differentiate between dissociative-PTSD and PTSD in rape and sexual assault victims?}

Armour, C., Elklit, A., Lauterbach, D., \& Elhai, J. D. (2014). The DSM-5 dissociative-PTSD subtype: Can levels of depression, anxiety, hostility, and sleeping difficulties differentiate between dissociative-PTSD and PTSD in rape and sexual assault victims? Journal of Anxiety Disorders, 28(4), 418-426.

https://doi.org/10.1016/j.janxdis.2013.12.008

Link to publication record in Ulster University Research Portal

Published in:

Journal of Anxiety Disorders

Publication Status:

Published (in print/issue): 01/01/2014

DOI:

10.1016/j.janxdis.2013.12.008

\section{Document Version}

Author Accepted version

\section{General rights}

Copyright for the publications made accessible via Ulster University's Research Portal is retained by the author(s) and / or other copyright owners and it is a condition of accessing these publications that users recognise and abide by the legal requirements associated with these rights.

\section{Take down policy}

The Research Portal is Ulster University's institutional repository that provides access to Ulster's research outputs. Every effort has been made to ensure that content in the Research Portal does not infringe any person's rights, or applicable UK laws. If you discover content in the Research Portal that you believe breaches copyright or violates any law, please contact pure-support@ulster.ac.uk. 
The DSM-5 dissociative-PTSD subtype: Can levels of depression, anxiety, hostility, and sleeping difficulties differentiate between dissociative-PTSD and PTSD in rape victims?

****CURRENTLY UNDER REVIEW WITH JOURNAL OF ANXIETY DISORDERS****

Authors;

Cherie Armour $^{{ }^{a} b_{*}}$; Ask Elklit ${ }^{\mathrm{b}}$; Dean Lauterbach ${ }^{\mathrm{c}}$, Jon D. Elhai ${ }^{\mathrm{d}}$

Author Affiliations;

${ }^{a}$ School of Psychology, University of Ulster at Coleraine Campus, Coleraine, Northern Ireland, UK

${ }^{b}$ The National Centre for Psychotraumatology, University of Southern Denmark, Odense, Denmark

${ }^{c}$ Department of Psychology, Eastern Michigan University, Michigan, USA

${ }^{\mathrm{d}}$ Department of Psychology and Department of Psychiatry, University of Toledo, Toledo, Ohio, USA

*Address correspondence to: Cherie Armour, School of Psychology, University of Ulster at Coleraine Campus, Cromore Road, Coleraine, Northern Ireland, United Kindgom, BT52 1SA; Email: armour.cherie@ gmail.com; Phone: (0044) 07876142643

Co-authors emails; Elklit: aelklit@ health.sdu.dk; Elhai: jonelhai@ gmail.com; Lauterbach: dlauterba@emich.edu 


\begin{abstract}
The DSM-5 currently includes a dissociative-PTSD subtype within its nomenclature. Several studies have confirmed the dissociative-PTSD subtype in both American Veteran and American civilian samples. Studies have begun to assess specific factors which differentiate between dissociative vs. non-dissociative PTSD. The current study takes a novel approach to investigating the presence of a dissociative-PTSD subtype in its use of European victims of sexual assault and rape $(N=351)$. Utilizing Latent Profile Analyses, we hypothesised that a discrete group of individuals would represent a dissociative-PTSD subtype. We additionally hypothesised that levels of depression, anger, hostility, and sleeping difficulties would differentiate dissociative-PTSD from a similarly severe form of PTSD in the absence of dissociation. Results concluded that there were four discrete groups termed baseline, moderate PTSD, high PTSD, and dissociative-PTSD. The dissociative-PTSD group encompassed $13.1 \%$ of the sample and evidenced significantly higher mean scores on measures of depression, anxiety, hostility, and sleeping difficulties. Implications are discussed in relation to both treatment planning and the newly published DSM-5.
\end{abstract}

Keywords: PTSD; Dissociation; Dissociative PTSD; Rape; Latent Profile Analysis; LPA 


\section{Introduction}

The fifth edition of the Diagnostic and Statistical Manual of Mental Disorders (DSM5; American Psychiatric Association [APA], 2013) was published in May 2013. Posttraumatic Stress Disorder (PTSD) has undergone a number of revisions, including the revision and addition of individual symptoms, a reorganisation of symptom groupings, and changes to the definition of the traumatic event which precedes the diagnosis (Elhai et al., 2012). Arguably, one of the most notable revisions to PTSD's nosology is the inclusion of a diagnostic subtype. Indeed, a diagnostic subtype of PTSD has never before been included within the DSM, despite a plethora of research noting that PTSD is highly comorbid with, and has been shown to have shared etiological origins to, a number of other psychiatric diagnoses including dissociation (Van der Hart, Van Ochten, Van Son, Steele, \& LensveltMulders, 2008). The current edition of the DSM; the DSM-5 includes a dissociative-PTSD subtype within its nosology.

Dissociation is currently defined as "an experienced loss of information or control over mental processes that, under normal circumstances, are available to conscious awareness, self-attribution, or control, in relation to the individual's age and cognitive development"(Cardena \& Carlson, 2011, p. 251). The idea that dissociation has a traumagenic etiology has been studied since the early twentieth century (Janet, 1907). A plethora of studies have since found support for the development of dissociative symptomatology in the wake of traumatic experiences (Gershuny \& Thayer, 1999). Notably, peri-traumatic dissociative experiences were formally included within the DSM-IV (APA, 1994) as part of the diagnostic criteria for Acute Stress Disorder (ASD), which was included within the nomenclature to account for the one month period between experiencing a trauma and receiving a formal diagnosis of PTSD. Thus ASD, and therefore dissociative experiences, are thought to account for early traumatic responding. Moreover, ASD is believed to be a 
reasonable predictor of future PTSD (Hansen, Armour, \& Elklit, 2012). Furthermore, a metaanalytic study highlighted that peri-traumatic dissociation was the strongest predictor of PTSD from a number of alternative known risk factors (Ozer, Best, Lipsey \& Weiss, 2003). Also notable, is that two PTSD symptoms specified in the DSM are considered dissociative: flashbacks and amnesia. However, given that peri-traumatic dissociation and persistent dissociation are phenomenologically distinct, occur at different times, and are weakly correlated (e.g., $r=.25$; Tichenor, Marmar, Weiss, Metzler \& Ronfeldt, 1996) it is pertinent to note that the DSM-5 included dissociative experiences of persistent dissociation; those of depersonalization and derealisation; thus the feeling of being disconnected from one's own emotions and body, and the feeling that the physical and interpersonal environment is strange or unfamiliar, therefore unreal (Carlson, Dalenberg \& McDade-Montez, 2012).

Despite certain commonalities between dissociative experiences and PTSD and a wealth of research supporting their association, the specific way in which the two forms of psychopathology co-occur is still contested within the literature. Indeed, a number of models attempting to explain the relationship between dissociation and PTSD have been proposed (see Dalenberg \& Carlson, 2012 for a review). The comorbidity model, which "posits that traumatic dissociation and PTSD are merely commonly co-occurring nosological entities sharing a necessary prerequisite broadly conceptualized as a traumatic event" (Simeon, 2007, p. 77), has largely been refuted. Dalenberg and colleagues reported that empirical findings are simply not consistent with the core components of the model. On this basis, empirical support for the model is lacking (Dalenberg \& Carlson, 2012). Models which are thought to accurately account for the relationship between dissociation and PTSD are subtype and component models. These models are similar in that both propose that dissociative experiences are a component of traumatic responding. Indeed, the component model proposes that dissociative experiences are associated with all components of PTSD (re-experiencing, 
avoidance/numbing, and arousal) and that both dissociative experiences and PTSD show a similar longitudinal course (i.e., both are increased immediately post-trauma but gradually decreases as the temporal proximity from trauma exposure decreases) (Dalenberg \& Carlson, 2012). Unique to the subtype model is the notion that levels of dissociation have the power to change the phenomenology of PTSD. In other words, individuals with high dissociation would be expected to report varying symptom patterns and severity levels of PTSD symptomatology. In addition, dissociative-PTSD would be expected to differentially correlate with external social and clinical covariates (Dalenberg \& Carlson, 2012).

Several early studies have investigated the relationship between dissociative experiences and PTSD using methods such as signal detection analysis, taxometric analysis, and the investigation of the distribution of scores; all of which have provided early support for the subtype model and thus the notion that only a subset of individuals with PTSD also report dissociative experiences (Ginzburg et al., 2006; Putnam et al., 1996; Waelde, Silvern \& Fairbank, 2005). Waelde et al. (2005) additionally reported that the subset of Vietnam Veterans whom reported dissociative PTSD within their study was also the subset who reported the most severe levels of PTSD.

More recent studies have implemented the techniques of Latent Class Analyses (LCA) or Latent Profile Analysis (LPA) (Hagenaars \& McCutcheon, 2002). Largely speaking these techniques are equivalent; however, the former is implemented with the use of categorical data whereas the latter is implemented with the use of continuous data. Both LCA and LPA uncover underlying latent classes (or groups) of individuals who respond in a similar manner to each other over a number of directly measured indicators. Simply put, LPA identifies various profiles based on qualitative (profile shape) and quantitative differences (symptom levels) in participant responding. Therefore, LCA and LPA have the ability to 
assess typological hypotheses and thus are ideally suited to researchers wishing to assess for a dissociative-PTSD subtype.

A seminal study was conducted by Wolf and colleagues (Wolf, Miller et al., 2012) using a sample of 492 veterans and their spouses. Participants were assessed for PTSD and dissociation via the Clinician Administered PTSD scale (CAPS; Blake et al., 1995). The LPA identified three underlying latent classes of participants: a small but distinct dissociative group, a high PTSD severity group, and a low PTSD severity group. As hypothesized the dissociative-PTSD group comprised only a subset (6\%) of the sample. In replication work, Wolf and colleagues (Wolf, Lunney et al., 2012) again conducted a LPA, this time on two different samples: 360 male veterans, assessed via the CAPS, and 284 female veterans, assessed via the Trauma Symptom Inventory. Three underlying latent classes were confirmed as optimal in both samples with a dissociative-PTSD subtype encompassing $15 \%$ of the all male sample and $30 \%$ of the all female sample.

Steuwe and colleauges (Steuwe, Lanius \& Frewen, 2012) implemented LPA using data from a civilian trauma sample $(N=134)$, who had predominatedly experienced sexual abuse, and who were assessed via the CAPS. In doing so, they revealed three latent classes, one of which was representative of a dissociative subtype and encompassed $25 \%$ of the sample. Slight methodological differences were apparent in Steuwe et al.'s study compared to those conducted by Wolf and colleagues (Wolf, Miller et al., 2012; Wolf, Lunney et al., 2012). For example, Steuwe et al. deliberatedly excluded the dissociative item of reduced awareness and thus based the analysis on only depersonalization and derealization items.

Additional support for the dissociative-PTSD subtype was reported by Armour and colleagues (Armour, Karstoft \& Richardson, in press), again using data gathered via the CAPS. Similar to Wolf and colleagues (Wolf, Miller et al., 2012; Wolf, Lunney et al., 2012), 
the LPA was conducted on a sample of veterans $(N=432)$; however on this occasion the veterans were Canadian rather than America. The LPA was conducted using the dissociative items of gaps in awareness, deprsonalization, and derealization. Notably, the LPA concluded that five, rather than three, underlying latent classes best represented the data. The dissociative subtype encompassed $13.7 \%$ of the sample. The authors highlighted that, irrespective of differences in the number of latent classes, the dissociative-PTSD subtype was strickingly similar to the dissociative-PTSD subtype found in earlier studies. Similar, in that it encompassed only a subset of individuals who reported a high level of PTSD symptoms and a high level dissociative experiences; in particular derealization and depersonalization, compared to alternative classes.

As aforementioned, the subtype model proposes that dissociative-PTSD would be expected to differentially correlate with external social and clinical covariates. LCA and LPA allow researchers to test hypotheses in relation to this as the latent classes can be used as the independent variable in a variety of statistical techniques. Wolf and colleagues assessed associations between their three classes and a number of demographic and trauma covariates. In comparing the dissociative-PTSD group to a group high on PTSD alone, they concluded that those in the dissociative group reported more childhood and adulthood experiences of sexual abuse (Wolf et al., 2012). In their replication study, Wolf and colleagues reported that they were unable to find any statistically significant differences in relation to demographics, trauma exposure, and Personality Disorders in the all male sample but that differences existed between the dissociative-PTSD and the PTSD alone group in the all female sample, with the dissociative- group reporting a higher rate of co-morbid PD, and a higher likelihood of selfidentifying their race as a racial minority. Notably, there were no differences related to level of exposure to sexual trauma (Wolf et al., 2012). 
Steuwe and colleagues (Steuwe et al., 2012) reported that individuals in their dissociative-PTSD group reported higher scores related to physical and sexual abuse, a greater number of current diagnoses, and greater comorbidity with major depression and specific phobia, compared to the high PTSD only group. Armour et al. (in press) reported that veterans were almost three times more likely $(\mathrm{OR}=2.733 ; \mathrm{CI}=1.253-5.967)$ to be categorized into the dissociative-PTSD group compared to the PTSD only group if they reported prior experiences of sexual assault (i.e., attempt to rape, made to perform any type of sexual act through force or threat of harm). No significant differences were found between the dissociative-PTSD group and the PTSD only group on a wide range of alternative traumas and a number of clinical covariates.

Previous research therefore suggests that traumas of a sexual nature may play a central role in the development of the dissociative subtype compared to a more 'pure' form of PTSD (i.e., in the absence of dissociative experiences) (Armour et al., in press; Ginzburg et al., 2006; Wolf et al., 2012). Conceptually this may make sense, given that traumatic experiences such as rape and sexual assault, which compromise the bodily integrity of the victim by an attacker, are regarded as significant risk factors for the development of pathological dissociation (Van Ommeren et al., 2001). If this is indeed the case, then a dissociative -PTSD subtype should be particularly evident in a sample of rape victims.

As noted above evidence in favor of the subtype model relating PTSD and dissociation is growing, however less is currently known in relation to factors which may predict the development of dissociative-PTSD rather than a more 'pure' form of PTSD. In summary, prior studies have assessed the role of a number of demographics, prior trauma experiences, clinical covariates (e.g., depression and anxiety), a greater number of current diagnoses, a greater comorbidity with major depression and specific phobia, and the presence of personality disorders (Armour et al., in press; Wolf, Miller et al., 2012; Wolf, Lunney et 
al., 2012). Studies have yet to assess whether differences exist between a dissociative-PTSD group and a PTSD only group in relation to scores on measures of hostility and sleeping difficulties, which are part of the PTSD construct. These covariates may be pertinent given previously reported associations between PTSD and sleep, (Mellman, David, Kulickbell, Hebding \& Nolan, 1995; Singareddy \& Balon, 2002) dissociation and sleep (cf. van der Kloet, Merckelbach, Giesbrecht \& Lynn, 2012; van der Kloet, Giesbrecht, Lynn, Merckelbach \& de Zutter, 2012), and hostility and anger with PTSD (cf. (Orth \& Wieland, 2006) and dissociation (Evren et al., 2013). Ultimately, suggesting the inter-relatedness of PTSD, dissociation, hostility, and sleeping difficulties.

\section{Aims and Hypotheses}

The aims of this study were two-fold; first we aimed to test if there was a homogeneous group of individuals who could be described in terms of a dissociative-PTSD subtype. In doing so we utilized LPA on 16 PTSD indicators from the Harvard Trauma Questionnaire (HTQ; Mollica et al., 1992) and three dissociative indicators from the Trauma Symptom Checklist (TSC; Briere \& Runtz, 1989) to objectively identify classes based on patterns of symptom endorsement across participants. Based on previous findings (Armour et al., in press; Steuwe et al., 2012; Wolf, Miller et al., 2012; Wolf, Lunney et al., 2012) we hypothesised that between three to five discrete groups would be uncovered; one of which would be characterised by the most severe levels of PTSD and dissociative symptomatology. Although we are cautious when hypothesising the expected prevalence of individuals in the dissociative-PTSD subgroup we did expect that it would be larger than that of previous reports given the noted associations between the dissociative-PTSD subtype and sexual traumas and given that the current sample encompasses victims of sexual assault and rape. The second aim of the study was to assess mean differences in covariate scores between the dissociative-PTSD class (should such a class have been uncovered) compared to alternative 
classes. The covariates of interest were anxiety, depression, hostility, and sleeping difficulties. Based on prior research we hypothesized that the dissociative-PTSD group would evidence higher mean scores in relation to depression and anxiety (Steuwe et al., 2012). Likewise, we tentatively hypothesized that the dissociative-PTSD group would evidence higher mean scores in relation to levels of hostility and sleeping difficulties. This hypothesis was based on previously noted associations between these covariates (cf., Evren et al., 2013; Mellman et al., 1995; Orth \& Wieland, 2006; van der Kloet et al., 2012) with PTSD and dissociation separately.

\section{Methods}

\subsection{Participants}

Participants were rape and sexual assault victims who had been in contact with a centre for rape victims (CRV) at the University Hospital of Aarhus, Denmark (Bramsen, Elklit \& Nielsen, 2009). All participants were initially assessed in relation to demographics and details of the assault were recorded on their first contact with the centre. Their early trauma related emotional responses were assessed between a few days to one week after their first contact with the CRV. Each participant was offered psychological counselling when in contact with the CRV. Participants were followed up at three months and completed a series of psychological measures. The current study focused on those who had been administered the Harvard Trauma Questionnaire and the Trauma Symptom Checklist $(N=351)$. However, 13 individuals were excluded as they were missing more than 3 HTQ items. Furthermore, as the current study chose to focus on females only, given the low rate of males within the sample, this resulted in the exclusion of an additional 25 individuals; 9 males and 16 individuals who failed to report their gender, leaving an effective sample size of 313 individuals. 


\subsection{Measures}

\subsubsection{The Harvard Trauma Questionnaire (HTQ; Mollica et al., 1992)}

The HTQ measures the presence and severity of PTSD based on a total of 30 items. Items are scored on a four point likert scale ranging from $1=$ not at all to $4=$ all the time. An individual's level of posttraumatic stress is indicated by summing the 30 individual items. The first sixteen items presented in the measure correspond to the 17 PTSD items as currently laid out in the DSM-IV-TR (APA, 2000). One question queries both physiological and psychological reactivity thus covers the DSM-IV-TR's B4 and B5 items. The scale has been previously reported as having good reliability and validity (Armour, Shevlin, Elklit \& Mroczek, 2012; Mollica et al., 1992). The reliability estimate of the scale, based on Cronbach's alpha, in the current study is .90 .

\subsubsection{The Trauma Symptom Checklist (TSC-33: Briere \& Runtz, 1989)}

The TSC-33 is a measure of psychological responses to wide array of traumatic experiences (Briere \& Runtz, 1989). Items are scored on a four point likert scale ranging from $1=$ not at all to $4=$ all the time. Symptoms can be combined to indicate symptom groups indicating depression, anxiety, dissociation, sleeping problems, suspicion of sexual abuse, somatization, interpersonal sensitivity, and hostility. In the current study, the reliability estimate of the scale based on Cronbach's alpha is .95 . The current study focused on the subscales of depression (.85), anxiety (.83), hostility (.74), and sleeping difficulties (.86) alpha estimates in parenthesis. The subscales and total scores gained from the TSC-33 have been previously reported as being internally consistent, with good discriminant validity (Briere \& Runtz, 1989). We also used three dissociative items from the dissociation subscale of the TSC to investigate the presence of a dissociative PTSD subtype. These items were 1. Loosing concentration / becoming absorbed; 2. Feelings of unrealness; and 3. Out of body 
Running head: DISSOCIATIVE SUBTYPE OF PTSD

experiences. The reliability alpha for the dissociation subscale of the TSC in the current study was .82 .

\subsection{Analytic Plan}

Descriptive statistics were explored using SPSS version 20. Latent Profile Analysis (LPA) was conducted using Mplus 6.12 software (Muthen \& Muthen, 1998-2012). LPA is an iterative exploratory statistical technique which allows for the identification of underlying latent subgroups within an overarching sample. The technique is exploratory as the number of underlying latent subgroups is not known apriori. We estimated models ranging from 2 and 6 latent classes using the default maximum likelihood with robust standard errors (MLR) estimator. Classification of individuals via LPA is based on individual mean scores (not posterior probabilities as in LCA) which indicate each individual cases membership in each class. Latent class models were compared across a number of fit statistics; The Aikake Information Criteria (AIC; Akaike, 1987), the Bayesian Information Criteria (BIC; Schwartz, 1978), the sample size adjusted Bayesian Information Criteria (ssBIC; Sclove, 1987) and the bootstrapped likelihood ratio test (BSLRT). Lower values on the AIC, BIC and ssBIC are indicative of better fit (Nylund, Nishina, Bellmore \& Graham, 2007; Nylund, Asparoutiov \& Muthen, 2007; Yang, 2006). Nylund et al. (2007) reported that the most reliable indicator of fit is the BIC; thus we focused our comparisons on this particular indicator. The BSLRT assesses whether a latent model with one additional class is superior to a latent model with one less class. Indeed, a non-significant BSLRT value $(p<.05)$ indicates that the latent model with one less class is the preferred option. We also consulted the Entropy (Ramaswamy, Desarbo, Reibstein \& Robinson, 1993) value as an indicator of classification quality within each individual model. Superior classification is indicated by values which approach 1 (Celeux \& Soromenho, 1996). 
After estimating the latent class distributions we assessed their relationship to covariates using pseudo-class draws. In other words, we did not implement a single step regression procedure whereby covariates were allowed to influence the latent class distributions. This decision was based on the fact that the formation of latent classes may be dependent on the specific covariates which are included within the model (Nylund et al., 2007). In using pseudo class draws we used the class variable as the independent variable in a one way between groups analyses of variance (ANOVA), in SPSS version 20, to determine if significant differences existed between classes in mean scores across the key external variables of depression, anxiety, hostility, and sleeping difficulties.

\section{Results}

\subsection{Effective sample characteristics}

The current sample $(N=313)$ was exclusively female. The mean age of participants was $22.38(S D=9.42)$. Over half of the effective sample were students $(64.5 \%), 18.6 \%$ were working, $7.2 \%$ were unemployed, 5.4\% were on sick leave / attending rehabilitation, and $4.3 \%$ were pensioned / retired. The majority of attacks were carried out by one offender $(93.4 \%)$ however $6.6 \%$ of victims were attacked by 2 or more offenders. Most victims knew their attackers; $62.2 \%$ identified their attackers as a friend or acquaintance, $8.5 \%$ identified their attackers as an ex romantic partner or spouse, $4.6 \%$ identified their attackers as a current partner, and $2.6 \%$ identified their attackers as a family member. Stranger attacks were reported by $20.2 \%$ of victims whereas $2.0 \%$ of victims reported that they were unable to identify their attacker. When categorised into known vs. unknown offenders, $77.9 \%$ of victims knew their attackers whereas $22.1 \%$ did not. A proportion of victims reported the use of alcohol or drugs when the event occurred; $44.7 \%$ reported being under the influence of alcohol, $0.3 \%$ reported being under the influence of drugs, and $4.5 \%$ reported being under the influence of both alcohol and drugs. Furthermore, $56.9 \%$ reported that they felt helpless and 
without power during the event, whereas $19.5 \%$ reported that they believed they were going to die.

\subsection{Latent Profile Analyses: Enumeration of classes}

The LPA was performed on the 16 HTQ items, corresponding to the 17 PTSD symptoms as specified in the DSM-IV-TR (APA, 2000), in addition to three dissociative items of 'loosing concentration / becoming absorbed', 'feelings of unrealness', and 'out of body experiences' from the dissociative subscale of the TSC. All latent class models of 2-6 latent classes successfully converged. Fit indices across all models are presented in Table 1. The BIC value, which has been shown to be the best indicator of model fit (Li \& Nyholt, 2001; Nylund et al., 2007; Rafferty, 1995), was lowest for the four class model. However, as we were cautious of the over-reliance on a single fit index to select the optimal model we explored the other model solutions more closely. Indeed, the $5 \mathrm{c}$ and $6 \mathrm{c}$ models both resulted in lower AIC and ssBIC values, however both also included classes comprised of a very small percentage of the overall sample. For example, the $5 \mathrm{c}$ model included a class of $8.6 \%$ of the sample and the $6 \mathrm{c}$ model included a class of only $6.2 \%$ of the sample. Small classes such as these may indicate the over-extraction of classes. More importantly however, on examining the profile plots it was clear that the additional class in the $5 \mathrm{c}$ and then $6 \mathrm{c}$ model was basically identical to that of an existing class with only slight quantitative differences. Furthermore, as outlined in Masyn (2013) we assessed the resultant latent classes of each of the models to ascertain which solution better helped us understand the phenomenon of interest, this was indeed the 4c model (see Fig. 1). Also following Masyn (2013), in selecting the $4 \mathrm{c}$ model as optimal over the $5 \mathrm{c}$ and the $6 \mathrm{c}$ models we did so by looking at the simplicity and clarity of each of the models. We felt that by selecting the $4 \mathrm{c}$ model we selected the most parsimonious option without losing any information. Notably, the Entropy value was highest for the $2 \mathrm{c}$ model and lowest for the $4 \mathrm{c}$ model. However, as Entropy is a measure of 
classification and not fit we did not use this for model selection, particularly given that all Entropy values exceeded .9 and models which approximate 1 are said to have clear classification. Therefore, based on fit statistics, clarity of meaning, parsimony, and visual inspections of profile plots, as suggested my Masyn (2013), the latent class model consisting of four classes was deemed optimal (see Table 1). The resultant profile plot can be seen in Figure 1. Table 2 presents the mean scores of items across each of the latent classes and the calculated difference between items for the dissociative-PTSD class and the High PTSD class when the difference exceeded 0.5 . Good discrimination between classes was revealed with the average latent class probabilities for most likely latent class membership being high $($ dissociative-PTSD class $=.96 ;$ high PTSD $=.92 ;$ moderate PTSD =.97; baseline $=.94)$.

****Insert Table, 1, Figure 1 and Table 2 approx. here please****

\subsection{ANOVA: Mean differences between groups across several covariates}

ANOVA was implemented to assess mean differences in scores across a number of variables as a function of latent class membership. The ANOVA was implemented using the four separate latent classes. The overall $\mathrm{F}$ test for each of the covariates was significant (overall $F[3,312]=147.98$ anxiety\}, $212.51\{$ depression $\}, 84.59\{$ hostility $\}$, and 83.46 \{sleeping difficulties\}, $P<.001$ for all covariates\}). Pairwise comparisons using Tukeys HSD revealed that all comparisons between all classes on scores related to anxiety, depression, hostility, and sleeping difficulties were significant. However, we were particularly interested in the mean differences in scores between the dissociative-PTSD and PTSD only classes. Indeed, pairwise comparisons revealed significant differences between the dissociative-PTSD group and the PTSD only group in relation to the TSC subscale scores of anxiety $(M=21.51 ; S D=4.68$ vs. $M=17.10 ; S D=3.85)$, depression $(M=29.63 ; S D=5$. 12 vs. $M=24.61 ; S D=4.06)$, hostility $(M=10.56 ; S D=2.92$ vs. $M=8.72 ; S D=2.35)$, and 
sleeping difficulties $(M=12.39 ; S D=3.18$ vs. $M=9.63 ; S D=3.09)$ respectively, (for all comparisons, $p<.001)$.

\section{Discussion}

The current study extends an emerging literature assessing typological hypotheses in relation to a dissociative-PTSD subtype. This investigation is timely given the recent publication of the DSM-5 (published in May 2013) and the inclusion of a dissociative-PTSD diagnostic subtype within its nosology. To our knowledge, this current investigation is the first to be conducted on a European sample, the first to base the investigation on non-military adult rape and sexual assault victims, and the first to assess a dissociative-PTSD subtype using the HTQ to measure PTSD and the TSC to measure dissociation. Furthermore, the current study extends previous work by assessing if a dissociative-PTSD subtype can be differentiated from a PTSD only group based on mean differences in depression, anxiety, hostility, and sleeping difficulties. Again this study is novel in its assessment of hostility and sleeping difficulties in relation to a dissociative-PTSD subtype.

The results of the LPA yielded further evidence in support of a dissociative-PTSD subtype. Indeed, we found four discrete classes based on participant's responding across 16 items measuring PTSD and three items measuring dissociation. Classes were termed baseline, moderate PTSD, high PTSD, and dissociative-PTSD. The baseline class was characterised by low mean scores across all PTSD and dissociative indicators. The moderate- and high-PTSD classes were quantitatively different in that the mean scores of all indicators were consistently higher in the moderate PTSD group and higher again in the high PTSD group. The dissociative-PTSD class evidenced the highest mean scores of all indicators and uniquely evidenced extremely high mean scores of the three dissociative indicators, particularly in relation to 'out of body experiences'. The dissociative-PTSD class also evidenced high scores 
on the recurrent dreams and reliving trauma indicators compared to the high PTSD class (see Table 2). Therefore, the current results support our hypothesis that a number of discrete groups would be uncovered; one of which would be characterised by the most severe levels of PTSD and dissociative symptomatology. Furthermore, this finding replicates that of previous studies which have found evidence of a dissociative-PTSD subtype (Armour et al., in press ; Steuwe et al., 2012; Wolf, Miller et al., 2012; Wolf, Lunney et al., 2012).

As previously noted, given discrepancies in the existing literature, we did not make a prediction in relation to the number of latent classes. Indeed, we found four discrete latent profiles rather than the previously reported three (Steuwe et al., 2012; Wolf, Miller et al., 2012; Wolf, Lunney et al., 2012) and five (Armour et al., in press) latent profiles. An additional discrepancy exists within the literature in relation to the prevalence of individuals within the dissociative-PTSD subtype. Previously the subtype has encompassed 6\% (Wolf et al., 2012), 15\% and 30\% (Wolf et al., 2012), 25\% (Steuwe et al., 2012) and 13.7\% (Armour et al., in press) of the total sample, whereas the dissociative-PTSD subtype in the current study encompasses $13.1 \%$ of the sample. We tentatively hypothesised that the current study may uncover a larger dissociative-PTSD subtype since participants were victims of sexual assault and rape and given the noted associations between traumas of a sexual nature and the development of the dissociative subtype compared to a more 'pure' form of PTSD (Armour et al., in press; Ginzburg et al., 2006; Wolf et al., 2012; Wolf et al., 2012). Furthermore, in attempting to explain why the dissociative-PTSD subtype encompassed $30 \%$ of the all female sample in their study, Wolf et al. (2012b) proposed that the "..high base rate of sexual trauma in the sample (93\%) led to an increased occurrence of dissociative phenomena" (p.686). Steuwe et al. (2012) stated that perhaps the subtype is larger than originally thought in predominately female samples accounting for between one third and one fourth of the sample. However, the current dissociative-PTSD subtype, found in our exclusively female 
sample of sexual assault and rape victims, encompassed $13.1 \%$ of the sample and so is contrary to our tentative hypothesis and to the proposals of others (cf. Steuwe et al., 2012; Wolf et al., 2012; Wolf et al., 2012).

The noted discrepancies in relation to number of classes and prevalence of individuals in the dissociative-PTSD class may be attributable to several factors. For example, the demographic and gender composition, the type and level of trauma exposure, the assessment and measurement procedures implemented, and the temporal proximity between trauma and assessment may vary greatly across studies. It is also pertinent to highlight that discrepancies in the prevalence of individuals found in the dissociative-PTSD subtype may be attributable to the number of extracted classes. Nevertheless, homogeneous groups of individuals who could be described in terms of a dissociative-PTSD subtype are being consistently reported (Armour et al., in press; Steuwe et al., 2012; Wolf et al., 2012; Wolf et al., 2012).

In line with the second aim of the current study; to explore whether certain covariates could differentiate between a dissociative-PTSD subgroup and a high PTSD only subgroup, prior research suggests that individuals in the dissociative-PTSD subtype may have a greater comorbidity pattern compared to those classified into non-dissociative PTSD groups. Indeed, the current study found significant differences between groups in relation to depression and anxiety, with those individuals reporting greater depressive and anxious symptomatology being found in the dissociative-PTSD subtype. These results are supportive of prior work. Indeed, Steuwe et al. (2012) reported greater comorbidity with Axis I disorders. Specifically, they reported that dissociative-PTSD individuals were more likely to report comorbidity with major depression and specific phobia. Wolf, Lunney et al. (2012) assessed comorbidity with PD and found that the female dissociative group reported a higher rate of co-morbid PD compared to non-dissociative groups. These findings provide further support for the subtype model discussed earlier; given that the subtype model proposes a change in PTSD's 
phenomenology for those in the dissociative subtype. A change in PTSD's phenomenology is indicated by higher comorbid disorders for those experiencing high vs. low or no dissociative symptoms (Steuwe et al., 2012). A greater pattern of comorbidity for those in the dissociative-PTSD subtype is however not fully conclusive as Wolf Lunney et al. (2012) reported no significant difference between groups on PD in the all male sample and Armour et al. (in press) found no significant differences between groups in relation to the clinical covariates of depression and anxiety in Canadian veterans. Notably the latter two studies were based almost exclusively on males. Thus, perhaps a greater pattern of comorbidity for those in the dissociative-PTSD subtype is evident predominately in females. Unfortunately, the current sample did not permit us to test this.

In an attempt to extend prior work we also assessed whether levels of hostility and sleeping difficulties were able to differentiate between the dissociative-PTSD subtype and non-dissociative groups. We hypothesized that these factors may be pertinent given previously noted associations between hostility and sleeping difficulties with PTSD and dissociation separately (Evren et al., 2013; Mellman et al., 1995; Orth \& Wieland, 2006; van der Kloet et al., 2012; van der Kloet et al., 2012) and with each other. With reference to the latter, difficulties with sleep, thus decreased length of sleep, has been shown to be associated with increased hostility (Ireland \& Culpin, 2006).

Individuals in the dissociative-PTSD group were found to be significantly more hostile than those in the PTSD only group. The concepts of hostility and anger are closely linked. Indeed, hostility is defined as an attitude in which individuals are mistrustful and disliking of others. In addition, individuals with a hostile attitude often believe that the behaviors of others are egoistic and hurtful. Having a hostile attitude in turn causes an increase in anger which is defined as an emotion characterized by many components; motivational, cognitive, behavioral, and physiological. Motivationally, an angry individual 
experiences increased hostile and aggressive impulses. Thus, one may reciprocally activate the other (Orth \& Wieland, 2006). Moreover, measures of hostility and anger often contain overlapping items and are thus highly correlated (cf. Eckhardt et al., 2004). Several empirical studies have focused explicitly on the relationship between anger and PTSD (cf. Orth \& Wieland, 2006) rather than on hostility and PTSD. Given the strong conceptual and empirical links we argue that the terms can be used interchangeably and thus suggest that the reported associations between anger and PTSD are equivalent to associations between hostility and PTSD. Indeed, this argument has been supported in a meta-analytic study of 39 empirical investigations on the topic (Orth \& Wieland, 2006).

Theoretically speaking the relationship between anger / hostility and PTSD may be based on an individual's attempt to avoid trauma-related memories and feelings of fear. The fear avoidance theory proposed by Foa and colleagues (Feeny et al., 2000) suggests that individuals shift their attention to anger rather than fear when experiencing intrusive recollections of the trauma as anger may intrinsically hold less negative valence for that individual. This is potentially counterproductive given that hostility and anger, as a means of avoiding trauma memories, may ultimately impede the emotional processing of trauma memories. Emotional processing theory suggests that the processing of the emotional content of trauma memories is directly related to treatment response (Hembree, Rauch \& Foa, 2003). This may explain why levels of PTSD and hostility are positively correlated. The question remains however as to why dissociative-PTSD groups may be more hostile / angry than pure PTSD groups over and above differences in PTSD severity. Particularly given that dissociative reactions to trauma seem quite distinct from reactions of anger and hostility. One commonality between dissociation and anger in relation to PTSD is that both are thought to serve as coping strategies which allow the individual to emotionally disengage from trauma memories (Feeny et al., 2000). Thus, it is possible that individuals in the dissociative-PTSD 
group, which is notably characterized by the severest levels of PTSD including that of intrusive recollections, attempt to emotionally disengage from their symptomatology by fluctuating between dissociative and hostile responses.

It is pertinent to note that traditionally anger and hostility were not regarded as central attributes of PTSD. However, the diagnostic criteria outlined in the DSM-IV included 'irritability and outbursts of anger' as an item within the hyperarousal symptom group. This item was retained within the DSM-5 diagnostic criteria. Previous work has questioned whether the association between anger and PTSD is simply a methodological artifact given that an item representing anger within the diagnostic criteria of PTSD has the potential to artificially inflate the correlation between measures of anger and thus hostility and PTSD. Two studies addressing exactly this issue have however reported that the relationship is not a methodological artifact (Novaco \& Chemtob, 2002; Orth \& Wieland, 2006). Ultimately, these results suggest that individuals who are regarded as hostile are at an increased risk for experiencing the dissociative-PTSD subtype.

PTSD is often associated with sleep disturbance (Singareddy \& Balon, 2002). Indeed, sleep disturbances / difficulties are part of PTSD's diagnostic criteria. Specifically, recurrent distressing dreams and difficulties with falling asleep are items found within PTSD's reexperiencing and arousal symptom groupings respectively ([DSM-5]; APA, 2013). Studies addressing sleep disturbances in individuals with PTSD have reported that insomnia, frequent startle or fear awakenings, sleep restlessness, trauma and non-trauma related nightmares, poor sleep quality, and REM sleep dysregulation are common (cf. Singareddy \& Balon, 2002). Furthermore, pre-trauma sleep disturbances, such as regular nightmares, have been shown to engender elevated post-traumatic responses (Mellman et al., 1995; Van der Kolk, Blitz, Burr, Sherry, \& Hartmann, 1984). Moreover, sleep focused treatment interventions are reported as being effective for reducing PTSD symptoms (Maher, Rego \& Asnis, 2006). 
The question remains, however, as to why sleep disturbances are more prominent in a dissociative-PTSD subtype compared to non dissociative PTSD groups as evident in the current study. As aforementioned, the idea that dissociation has a traumagenic aetiology has been extensively studied and supported (Bremner, 1999; Spiegel et al., 2011). Indeed, the perspective that dissociative symptoms develop as a coping response to psychological trauma has been termed the posttraumatic model (PTM) of dissociation. In particular, the model proposes that dissociative responses are ultimately mental strategies which are employed to avoid painful traumatic memories (van der Kloet et al., 2012). Researchers have recently suggested that given the links between dissociative phenomenon and sleep related experiences, perhaps sleep related experiences may play an important role in the trauma dissociation relationship. This proposal is attributable to a number of studies showing that negative sleep experiences, such a nightmares and fear awakening are related to dissociative symptomatology. Indeed, the estimated correlation between negative sleep disturbances and dissociative symptomatology falls between .30 and .55 (based on an excess of 5,600 participants; van der Kloet et al., 2012). Furthermore, studies have shown that sleep normalization interventions are effective at reducing dissociative symtpomotology (van der Kloet et al., 2012).

Ultimately, findings suggest that sleep disturbances are prominent features of both PTSD and dissociation. One pertinent line of research which may elucidate the sleep and dissociative-PTSD link is that related to the individual associations between sleep, dissociation, and PTSD with memory fragmentation. In particular, it has been reported that sleep loss may create memory fragmentation, given that sleep loss interferes with the consolidation and encoding of memory (cf. Harrison \& Horne, 2000). The fragmentation of trauma memories has long been implicated in the development of posttraumatic symptomatology. Indeed, people experiencing PTSD are thought to have trauma memories 
which are difficult to recall, poorly organised, and have issues with the temporal ordering of events (Elhers \& Clark, 2000). Interestingly, memory fragmentation is thought to occur due to peri-traumatic dissociation, in that dissociation at the time of the trauma results in only partial storage of the memory. Moreover, continued dissociation further inhibits processing and elaboration of the traumatic memory and thus maintains fragmentation of such memories (cf., Bedard-Gilligan \& Zoellner, 2012). Thus, the interrelatedness of sleep, dissociation, and PTSD with the fragmentation of trauma memories may account for why individuals in the dissociative-PTSD subtype report greater problems with sleep, in that these individuals also experience the greatest issues with traumatic memory fragmentation.

\subsection{Conclusion}

Consistent with the extant literature, the current study provides further evidence for a discrete dissociative-PTSD subtype which is apparent in only a subset of PTSD inflicted individuals. Contrary to previous studies utilizing LCA or LPA we uncovered a total of 4 rather than 3 or 5 latent classes (Armour et al., in press; Steuwe et al., 2012; Wolf et al., in press; Wolf et al., 2012). Furthermore, our dissociative-PTSD class encompassed $13.1 \%$ of the sample which is lower than would have been expected based on prior reported associations with the dissociative subtype, females, and traumas of a sexual nature (Armour et al., in press; Steuwe et al., 2012). Notwithstanding these discrepancies, the dissociativePTSD subtype we uncovered was once again remarkably similar to that found in previous studies; in that the levels of PTSD symptoms in the dissociative-PTSD class were almost identical to those in the severe PTSD class but that individuals in the dissociative-PTSD class uniquely evidenced extremely high mean scores on dissociative indicators. Thus, a pertinent line of enquiry relates to factors which may differentiate between a dissociative-PTSD group and a severe (more pure) PTSD group. In the current study patterns of comorbidity were shown to be elevated in the dissociative-PTSD subtype compared to the severe PTSD only 
subgroup, in relation to depression and anxiety. This is supportive of previous findings (Steuwe et al., 2012; Wolf et al., 2012) based on female subsamples but not fully conclusive given contrary findings (Armour et al., in press; Wolf et al., 2012b from male subsamples. The current study further concluded that hostility and sleeping difficulties were more prominent in the dissociative-PTSD subtype. The former perhaps attributable to dissociativePTSD individuals attempting to emotionally disengage from their symptomatology (Feeny et al., 2000) by fluctuating between dissociative and hostile responses and the latter attributable to the interrelatedness of sleep, dissociation, and PTSD with the fragmentation of trauma memories (van der Kloet et al., 2012).

Taken together with extant findings, the current results support the subtype model in relation to dissociative-PTSD and thus ultimately support the inclusion of a dissociativePTSD subtype into the DSM-5 nomenclature. Including a dissociative-PTSD subtype within the diagnostic criteria increases clinician awareness about the potential presence of dissociative symptoms in individuals suffering from PTSD. In turn this can inform assessment and treatment approaches. Furthermore, investigations into important predictors of dissociative-PTSD can elucidate risk and resilience for the same. For example, individuals with PTSD who present with high levels of hostility and severe sleeping difficulties may additionally be presenting with dissociative symptomatology. Again, this informs assessment and treatment planning. Notably, further research is required to determine how each of the constructs is temporally related. Questions remain in relation to whether sleeping difficulties and hostility, as associated with PTSD, result in dissociative symptomatology or whether dissociative symptomatology combined with PTSD increases sleeping difficulties and hostility? It is also possible that sleeping difficulties increase hostility or indeed that they reciprocally activate each other. Longitudinal studies are needed to shed further light on such questions. Furthermore, both PTSD and dissociation have been shown to have many 
correlates; how each of these correlates relates specifically to dissociative-PTSD is an avenue for future research.

\subsection{Limitations}

A number of limitations must be acknowledged. First, the assessment of a dissociative-PTSD subtype was based on self-report measures and on a restricted range of dissociative indicators. Ideally, further investigation into the dissociative-PTSD subtype would use a full measure of dissociation such as the Dissociative Experiences Scale (Bernstein \& Putman, 1986) rather than using items from a sub-scale which measures general posttraumatic responding. Furthermore, our trauma sample is particularly focused in that our sample was exclusively female and all participants had experienced sexual assault and rape. This is however a novel aspect of our study as prior work has tended to focus on combat veterans. Notably, no study to date has investigated the dissociative-PTSD subtype in an epidemiological sample with heterogeneous trauma experiences. An investigation of this type would add significantly to the extant knowledge given that population based samples cover a random sample of those affected in the population thus cover the full spectrum of severity. Moreover, results obtained are representative of, thus generalisable to, the population rather than to a particular setting or group, e.g., rape victims or combat veterans. Notwithstanding these limitations the current study supports and extends the extant evidence related to a dissociative-PTSD subtype, which was recently included in the nomenclature of PTSD within the DSM-5. 


\section{References}

Akaike, H. (1987). Factor analysis and AIC. Psychometrika, 52, 317-332. doi: $10.1007 / \mathrm{bf02294359}$

American Psychiatric Association. (1882). Diagnostic and Statistical Manual of Mental Disorders. (3rd ed.). Washington, DC: Author.

American Psychiatric Association (1987). Diagnostic and Statistical Manual of Mental Disorders (3rd ed., text rev.) Washington, DC: Author.

American Psychiatric Association (2000). Diagnostic and Statistical Manual of Mental Disorders (4th ed., text rev.). Washington, DC: Author.

Armour, C., Karstoft, K. I., \& Richardson, J. D. (2013). The co-occurrence of PTSD and dissociation: Differentiating severe PTSD from dissociative PTSD. Submitted Manuscript

Armour, C., Shevlin, M., Elklit, A., \& Mroczek, D. (2012). A latent growth mixture modelling approach to PTSD in rape victims. Traumatology, 18, 20-28.

Bedard-Gilligan, M., \& Zoellner, L. A. (2012). Dissociation and memory fragmentation in post-traumatic stress disorder: An evaluation of the dissociative encoding hypothesis. Memory, 20, 277-299. doi: 10.1080/09658211.2012.655747

Bernstein, E. M., \& Putnam, F. W. (1986). Development, reliability, and validity of a dissociation scale. Journal of Nervous \& Mental Disease, 174, 72735. doi:10.1097/00005053-198612000-00004 
Blake, D. D., Weathers, F. W., Nagy, L. M., Kaloupek, D. G., Gusman, F. D., Charney, D. S., \& Keane, T. M. (1995). The development of a clinician-administered PTSD scale. Journal of Trauma Stress, 8, 75-90.

Bramsen, R. H., Elklit, A. \& Nielsen, L. H. (2009). A Danish Model for Treating Rape Victims - A Multidisciplinary Public Approach. The Journal of Aggression, Maltreatment, and Trauma, 18, 886-905.

Bremner, J. D. (1999). Acute and chronic responses to psychological trauma: Where do we go from here? American Journal of Psychiatry, 156, 349-51.

Briere, J., \& Runtz, M. (1989). The trauma symptom checklist (TSC-33): Early data on a new scale. Journal of Interpersonal Violence, 4, 151-163.

Cardena, E., \& Carlson, E. (2011). Acute stress disorder revisited. Annual Review of Clinical Psychology, 7, 245-267. doi: 10.1146/annurev-clinpsy-032210-104502

Carlson, E. B., Dalenberg, C., \& McDade-Montez, E. (2012). Dissociation in posttraumatic stress disorder part I: Definitions and review of research. Psychological Trauma: Theory, Research, Practice, and Policy, No Pagination Specified.

Celeux, G., \& Soromenho, G. (1996). An entropy criterion for assessing the number of clusters in a mixture model. Journal of Classification, 13, 195-212.

Dalenberg, C., \& Carlson, E. B. (2012). Dissociation in posttraumatic stress disorder part II: How theoretical models fit the empirical evidence and recommendations for modifying the diagnostic criteria for PTSD. Psychological Trauma-Theory Research Practice and Policy, 4, 551-559. doi: 10.1037/a0027900 
Elhai, J. D., Miller, M. E., Ford, J. D., Biehn, T. L., Palmieri, P. A., \& Frueh, B. C. (2012). Posttraumatic stress disorder in DSM-5: Estimates of prevalence and symptom structure in a nonclinical sample of college students. Journal of Anxiety Disorders, 26, 58-64. doi: 10.1016/j.janxdis.2011.08.013

Evren, C., Cinar, O., Evren, B., Ulku, M., Karabulut, V., \& Umut, G. (2013). The mediator roles of trait anxiety, hostility, and impulsivity in the association between childhood trauma and dissociation in male substance-dependent inpatients. Comprehensive Psychiatry, 54, 158-166. doi: 10.1016/j.comppsych.2012.06.013

Feeny, N. C., Zoellner, L. A., \& Foa, E. B. (2000). Anger, dissociation, and posttraumatic stress disorder among female assault victims. Journal of Traumatic Stress, 13, 89-100. doi: 10.1023/A:1007725015225

Gershuny, B. S., \& Thayer, J. F. (1999). Relations among psychological trauma, dissociative phenomena, and trauma-related distress: A review and integration. Clinical Psychology Review, 19, 631-657. doi: 10.1016/S0272-7358(98)00103-2

Ginzburg, K., Koopman, C., Butler, L. D., Palesh, O., Kraemer, H. C., Classen, C. C., \& Spiegel, D. (2006). Evidence for a dissociative subtype of post-traumatic stress disorder among help-seeking childhood sexual abuse survivors. Journal of Trauma \&Dissociation, 7, 7-27. doi: 10.1300/J229v07n02_02

Hagenaars, J. A., \& McCutcheon, A. L. (Eds.). (2002). Applied latent class analysis. Cambridge: Cambridge University Press. 
Running head: DISSOCIATIVE SUBTYPE OF PTSD

Hansen, M., Armour, C., \& Elklit, A. (2012). Assessing a dysphoric arousal model of acute stress disorder symptoms in a clinical sample of rape and bank robbery victims. European Journal of Psychotraumatology, 3 doi: 10.3402/ejpt.v3i0.18201

Harrison, Y., \& Horne, J. A. (2000). Sleep loss and temporal memory. Quarterly Journal of Experimental Psychology Section A-Human Experimental Psychology, 53, 271-279. doi: $10.1080 / 027249800390772$

Hembree, E. A., Rauch, S. A. M., \& Foa, E. B. (2003). Beyond the manual: The insider's guide to prolonged exposure therapy for PTSD. Cognitive and Behavioral Practice, 10, 22-30. doi: 10.1016/S1077-7229(03)80005-6

Ireland, J. L., \& Culpin, V. (2006). The relationship between sleeping problems and aggression, anger, and impulsivity in a population of juvenile and young offenders. Journal of Adolescent Health, 38, 649-655. doi: 10.1016/j.jadohealth.2005.05.027

Janet, P. (1907). The major symptoms of hysteria. New York: McMillan.

Li, W., \& Nyholt, D. R. (2001). Marker selection by Akaike information criterion and Bayesian information criterion. Genetic Epidemiology, 21, S272-S277

Maher, M. J., Rego, S. A., \& Asnis, G. M. (2006). Sleep disturbances in patients with posttraumatic stress disorder - epidemiology, impact and approaches to management. Cns Drugs, 20, 567-590. doi: 10.2165/00023210-200620070-00003

Masyn, K. E. (2013). Latent class analysis and finite mixture modeling. In T. D. Little (Ed.), The Oxford Handbook of Quantitative Methods (vol 2.) (pp. 551-611). 
Mellman, T. A., David, D., Kulickbell, R., Hebding, J., \& Nolan, B. (1995). Sleep disturbance and its relationship to psychiatric morbidity after hurricane-andrew. American Journal of Psychiatry, 152, 1659-1663.

Mollica, R. F., Caspi-Yavin, Y., Bollini, P., Truong, T., Tor, S., \& Lavelle, J. (1992). The harvard trauma questionnaire. validating a cross-cultural instrument for measuring torture, trauma, and posttraumatic stress disorder in indochinese refugees. The Journal of Nervous and Mental Disease, 180, 111-116.

Muthen, L. K., \& Muthen, B. O. (1998-2012). Mplus user's guide (Seventh Edition ed.). Los Angeles, CA:

Novaco, R. W., \& Chemtob, C. M. (2002). Anger and combat-related posttraumatic stress disorder. Journal of Traumatic Stress, 15, 123-132. doi: 10.1023/A:1014855924072

Nylund, K., Nishina, A., Bellmore, A., \& Graham, S. (2007). Subtypes, severity, and structural stability of peer victimization: What does latent class analysis say? Child Development, 78, 1706-1722.

Nylund, K. L., Asparoutiov, T., \& Muthen, B. O. (2007). Deciding on the number of classes in latent class analysis and growth mixture modeling: A monte carlo simulation study. Structural Equation Modeling-a Multidisciplinary Journal, 14, 535-569.

Orth, U., \& Wieland, E. (2006). Anger, hostility, and posttraumatic stress disorder in traumaexposed adults: A meta-analysis. Journal of Consulting and Clinical Psychology, 74, 698-706. doi: 10.1037/0022-006X.74.4.698 
Ozer, E. J., Best, S. R., Lipsey, T. L., \& Weiss, D. S. (2003). Predictors of posttraumatic stress disorder and symptoms in adults: A meta-analysis. Psychological Bulletin, 129, 52-73. doi: Doi 10.1037//0033-2909.129.1.52

Putnam, F. W., Carlson, E. B., Ross, C. A., Anderson, G., Clark, P., Torem, M., . . Braun, B. G. (1996). Patterns of dissociation in clinical and nonclinical samples. Journal of Nervous \& Mental Disease, 184, 673-9.

Raftery, A. E. (1995). Bayesian model selection in social research. In: P.V. Marsden (Ed.), Sociological Methodology. Cambridge, MA: Blackwell

Ramaswamy, V., Desarbo, W. S., Reibstein, D. J., \& Robinson, W. T. (1993). An empirical pooling approach for estimating marketing mix elasticities with PIMS data. Marketing Science, 12, 103-124.

Schwartz, G. (1978). Estimating the dimensions of a model. Annuals of Statistics, 6, 461-464.

Sclove, S. L. (1987). Application of model-selection criteria to some problems in multivariate-analysis. Psychometrika, 52, 333-343.

Simeon, D. (2007). Relationships between dissociation and posttraumatic stress disorder. Traumatic dissociation: Neurobiology and treatment (pp. 77-101). Arlington, VA: American Psychiatric Publishing, Inc ; US.

Singareddy, R. K., \& Balon, R. (2002). Sleep in posttraumatic stress disorder. Annals of Clinical Psychiatry : Official Journal of the American Academy of Clinical Psychiatrists, 14, 183-90. doi: 10.3109/10401230209147455 
Spiegel, D., Loewenstein, R. J., Lewis-Fernandez, R., Sar, V., Simeon, D., Vermetten, E., Dell, P. F. (2011). Dissociative disorders in DSM-5. Depression and Anxiety, 28, E17E45. doi: 10.1002/da.20923

Steuwe, C., Lanius, R. A., \& Frewen, P. A. (2012). Evidence for a dissociative subtype of ptsd by latent profile and confirmatory factor analyses in a civilian sample. Depression and Anxiety, 29, 689-700. doi: 10.1002/da.21944

van der Hart, O., van Ochten, J. M., van Son, M. J., Steele, K., \& Lensvelt-Mulders, G. (2008). Relations among peritraumatic dissociation and posttraumatic stress: A critical review. Journal of Trauma \& Dissociation, 9, 481-505.

van der Kloet, D., Giesbrecht, T., Lynn, S. J., Merckelbach, H., \& de Zutter, A. (2012). Sleep normalization and decrease in dissociative experiences: Evaluation in an inpatient sample. Journal of Abnormal Psychology, 121, 140-150. doi: 10.1037/a0024781

van der Kloet, D., Merckelbach, H., Giesbrecht, T., \& Lynn, S. J. (2012). Fragmented sleep, fragmented mind: The role of sleep in dissociative symptoms. Perspectives on Psychological Science, 7, 159-175. doi: 10.1177/1745691612437597

Van der Kolk, B., Blitz, R., Burr, W., Sherry, S., \& Hartmann, E. (1984). Nightmares and trauma a comparison of nightmares after combat with lifelong nightmares in veterans. American Journal of Psychiatry, 141, 187-190.

Van Ommeren, M., Sharma, B., Komproe, I., Poudyal, B. N., Sharma, G. K., Cardena, E., \& De Jong, J. T. V. M. (2001). Trauma and loss as determinants of medically unexplained epidemic illness in a bhutanese refugee camp. Psychological Medicine, 31, 1259-1267. doi: $10.1017 / \mathrm{S} 0033291701004470$ 
Waelde, L. C., Silvern, L., \& Fairbank, J. A. (2005). A taxometric investigation of dissociation in Vietnam Veterans. Journal of Traumatic Stress, 18, 359-69. doi: $10.1002 /$ jts. 20034

Wolf, E. J., Miller, M. W., Reardon, A. F., Ryabchenko, K. A., Castillo, D., \& Freund, R. (in press). A latent class analysis of dissociation and PTSD: Evidence for a dissociative subtype. Archives of General Psychiatry,

Wolf, E. J., Lunney, C. A., Miller, M. W., Resick, P. A., Friedman, M. J., \& Schnurr, P. P. (2012). The dissociative subtype of PTSD: A replication and extension. Depression and Anxiety, 29, 679-688. doi: 10.1002/da.21946

Wolf, E. J., Miller, M. W., Reardon, A. F., Ryabchenko, K. A., Castillo, D., \& Freund, R. (2012). A latent class analysis of dissociation and posttraumatic stress disorder evidence for a dissociative subtype. Archives of General Psychiatry, 69, 698-705.

Yang, C. C. (2006). Evaluating latent class analysis models in qualitative phenotype identification. Computational Statistics \& Data Analysis, 50, 1090-1104. doi: 10.1016/J.Csda.2004.11.004 
Running head: DISSOCIATIVE SUBTYPE OF PTSD

Figure 1.

Four-class Latent Profile Plot of PTSD and Dissociative Indicators.

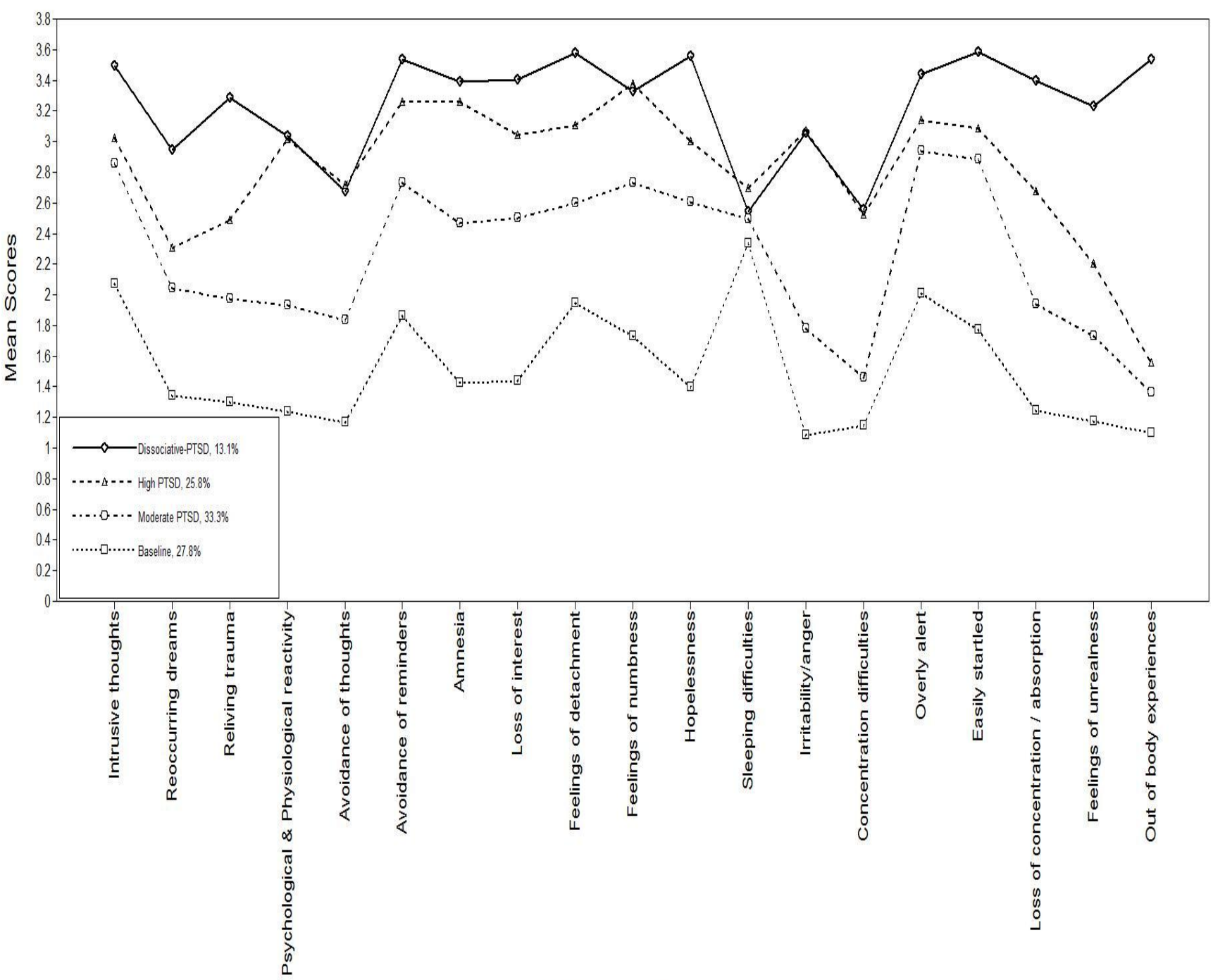

PTSD / Dissociative ltems 
Running head: DISSOCIATIVE SUBTYPE OF PTSD

Table 1.

Fit of Competing 2-6 Class Models

\begin{tabular}{llllll}
\hline & AIC & BIC & ssBIC & Entropy & BSLRT (p) \\
\hline 2C & 15418.574 & 15635.854 & 15451.896 & 0.941 & 1750.233 \\
& & & & & 0.000 \\
3C & 14989.318 & 15281.522 & 15034.131 & 0.911 & 469.256 \\
& & & & & 0.000 \\
4C & 14797.587 & 15164.715 & 14853.890 & 0.906 & 231.732 \\
& & & & & 0.000 \\
5C & 14740.724 & 15182.776 & 14808.517 & 0.911 & 96.863 \\
& & & & & 0.000 \\
& & & & & \\
6C & 14685.698 & 15202.674 & 14764.981 & 0.905 & 95.125 \\
& & & & 0.000 \\
\hline
\end{tabular}

Note. AIC $=$ Akaike information criterion; $\mathrm{BIC}=$ Bayesian Information Criterion; SSABIC $=$ sample size adjusted Bayesian Information Criterion; BSLRT $(p)=2$ times the Loglikelihood difference and associated $p$ value 
Running head: DISSOCIATIVE SUBTYPE OF PTSD

Table 2. Latent class indicator items mean scores across latent classes

\begin{tabular}{|c|c|c|c|c|}
\hline $\begin{array}{l}\text { Indicator Mean Score / Latent } \\
\text { Class }\end{array}$ & $\begin{array}{l}\text { Dissociative-PTSD (13.1\%) } \\
\text { M (diff with high PTSD if } \\
\text { exceeds .05) }\end{array}$ & $\begin{array}{l}\text { High PTSD } \\
(25.8 \%)\end{array}$ & $\begin{array}{c}\text { Moderate PTSD } \\
(33.3 \%)\end{array}$ & $\begin{array}{l}\text { Baseline } \\
(27.8 \%)\end{array}$ \\
\hline Intrusive Thoughts & 3.497 & 3.021 & 2.856 & 0.384 \\
\hline Recurring Dreams & $2.948 \quad(0.639)$ & 2.309 & 2.045 & 0.518 \\
\hline Reliving Trauma & $3.284 \quad(0.800)$ & 2.484 & 1.972 & 0.719 \\
\hline $\begin{array}{c}\text { Psychological/physiological } \\
\text { Reactivity }\end{array}$ & 3.039 & 3.016 & 1.932 & 0.518 \\
\hline Avoidance of thoughts & 2.674 & 2.715 & 1.835 & 0.585 \\
\hline Avoidance of reminders & 3.536 & 3.258 & 2.733 & 0.731 \\
\hline Amnesia & 3.388 & 3.260 & 2.464 & 0.456 \\
\hline Loss of interest & 3.404 & 3.040 & 2.502 & 0.846 \\
\hline Feelings of detachment & 3.581 & 3.104 & 2.595 & 0.661 \\
\hline
\end{tabular}


Running head: DISSOCIATIVE SUBTYPE OF PTSD

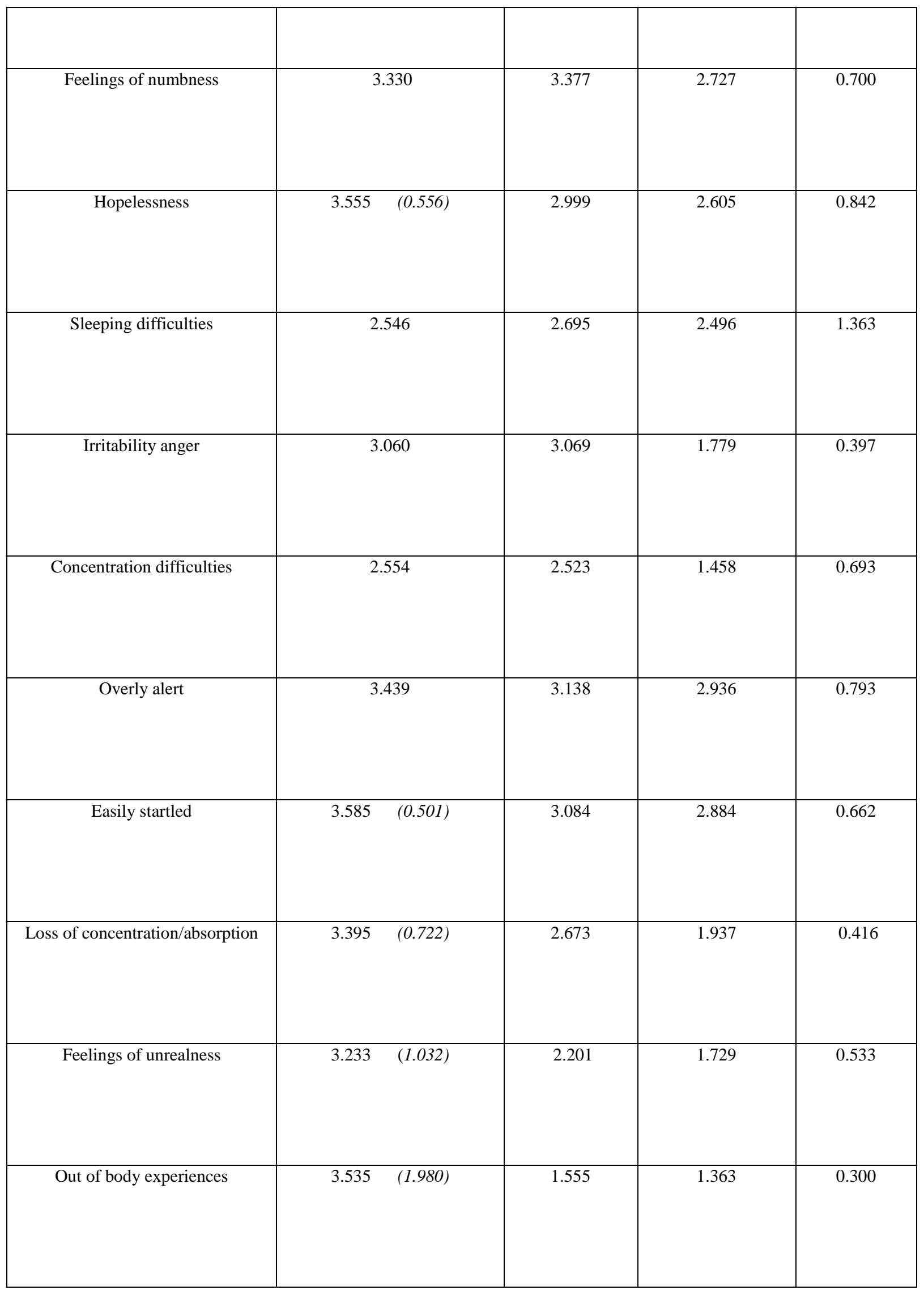


Running head: DISSOCIATIVE SUBTYPE OF PTSD 